\title{
One-Pot, Three-Component Synthesis of Novel 4-Phenyl-2-[3-(alkynyl/ alkenyl/aryl)phenyl]pyrimidine Libraries via Michael Addition, Cyclization, and C-C Coupling Reactions: A New MCR Strategy
}

\author{
L. Srinivasula Reddy, ${ }^{\mathrm{a}, \mathrm{b}}$ T. Ram Reddy, ${ }^{\mathrm{b}}$ N. C. Gangi Reddy, ${ }^{\text {c }}$ Reddy Bodireddy Mohan, ${ }^{\mathrm{c}}$ Y. Lingappa ${ }^{\mathrm{a}}$ \\ a Department of Chemistry, Sri Venkateswara University, Tirupati 517 502, Andhra Pradesh, India \\ b Dr. Reddy's Laboratories Limited, Bollaram Road, Miyapur, Hyderabad 500 049, Andhra Pradesh, India \\ c Department of Chemistry, School of Physical Sciences, Yogi Vemana University, Kadapa 516 003, Andhra Pradesh, India \\ E-mail: ncgreddy@yogivemanauniversity.ac.in
}

Received: 11.10.2012; Accepted: 30.10.2012

\begin{abstract}
Privileged medicinal scaffolds based on the structures of 4-phenyl-2-[3-(alkynyl/alkenyl/aryl)phenyl]-substituted pyrimidines have been synthesized via a single-step, three-component reaction of 3-(dimethylamino)-1-phenylprop-2-en-1-one (enaminone), 3-bromobenzimidamide hydrochloride, and various alkynes/alkenes/arylboronic acids. The mechanism of this multicomponent reaction (MCR) involves a Michael addition, cyclization, isomerization, and dehydration, followed by Sonogashira, Heck or Suzuki coupling. This new MCR strategy afforded a new compound library based on pyrimidine framework.
\end{abstract}

Key words: multi-component reactions (MCRs), Michael addition, Pd catalyst, $\mathrm{C}-\mathrm{C}$ coupling reactions

The synthesis of 'privileged medicinal scaffolds' is highly important as these compounds often act as ligands for a number of functionally and structurally diverse biological receptors and further serve as a platform for developing pharmaceutical agents for diverse applications. ${ }^{1}$ For instance, pyrimidine and its derivatives are considered as 'privileged scaffold' due to their potential biological activities such as antihypertensive, ${ }^{2}$ antipyretic, ${ }^{3}$ antibacteri$\mathrm{al}^{4,6}$ antifungal ${ }^{7,8}$ anticancer,,${ }^{9,10}$ anti-inflammatory, ${ }^{11,12}$ and cardio-protective activities. ${ }^{13}$ The structural features of pyrimidines are also found in some pesticides,,${ }^{14}$ herbicides, and plant growth regulators. ${ }^{15}$ Consequently, methodologies for the synthesis of novel pyrimidines or pyrimidine-fused compounds are of particular interest in the medicinal and agrochemical research areas. ${ }^{16,17}$

These vast applications have inspired the development of a number of methods for the preparation of pyrimidine de- rivatives. ${ }^{18}$ In addition to reports about the variation of established protocols, new methods ${ }^{18}$ were also described on the union of amine and carbonyl-containing fragments and $N$-vinyl, $N$-aryl amides and nitriles to gather the imperative pyrimidine substructures. Additionally, the advancement of transition metal-catalyzed methodologies for cross-coupling of activated azaheterocycles offer complementary access to substituted azaheterocycles. ${ }^{19}$ However, literature studies reveal that, none has been reported on the synthesis of 4-phenyl-2-[3-(alkynyl/alkenyl/ aryl)phenyl]-substituted pyrimidine derivatives from enaminone, 3-bromobenzimidamide hydrochloride, and various alkynes/alkenes/arylboronic acids via a single step, one-pot multi-component reaction. Multi-component reactions (MCRs) are powerful strategies for the quick synthesis of diverse and complex organic molecules of potential interest particularly in the area of material science and drug discovery. ${ }^{20}$ MCRs have attracted much attention owing to their excellent synthetic efficiency, intrinsic atom economy, high selectivity, procedural simplicity, and environmental friendliness. ${ }^{20 \mathrm{a}, 21}$ As a result, the search and discovery of new MCR have attained significant value. ${ }^{22}$

Herein we report a new MCR strategy for the synthesis of 4-phenyl-2-[3-(alkynyl/alkenyl/aryl)phenyl]-substituted pyrimidine derivatives from the reaction of enaminone 1 , 3 -bromobenzimidamide hydrochloride (2), and various alkynes $\mathbf{3}$, alkenes 5 or arylboronic acids $\mathbf{7}$ in the presence of $\mathrm{K}_{2} \mathrm{CO}_{3}$ and Pd catalyst in DMF (Scheme 1).
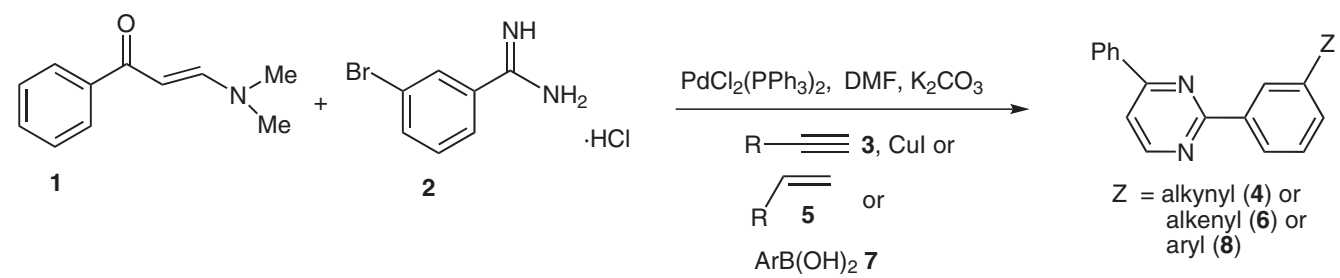

Scheme 1 One-pot three-component synthesis of 4-phenyl-2-[3-(alkynyl/alkenyl/aryl)phenyl]-substituted pyrimidines

SYNTHESIS 2013, 45, 0075-0084

Advanced online publication: 27.11.2012

DOI: 10.1055/s-0032-1316814; Art ID: SS-2012-N0802-OP

(C) Georg Thieme Verlag Stuttgart · New York 
In the present study, our initial objective was to identify well-suited reaction conditions for MCR besides seeking an appropriate Pd catalyst, solvent, and base for the construction of pyrimidine scaffold followed by Sonogashira/ Heck/Suzuki coupling in a single step operation. We choose to assess the pyrimidine formation/Sonogashira coupling initially for this purpose. Accordingly, few palladium catalysts, solvents, and bases were examined to determine the effect on the course of MCR of enaminone 1, 3-bromobenzimidamide hydrochloride (2), and prop-2yn-1-ol (3a) in the presence of CuI (Table 1) as a model reaction. Initially, the reaction carried out by using $10 \%$ $\mathrm{Pd} / \mathrm{C}$ catalyst with $\mathrm{K}_{2} \mathrm{CO}_{3}$ in $\mathrm{DMF}$ at $100{ }^{\circ} \mathrm{C}$ for 24 hours afforded the product $\mathbf{4 a}$ in $10 \%$ yield (Table 1, entry 1 ). Later, optimization of the reaction conditions was scrutinized to increase the yield of the product. Towards this direction, a variety of $\mathrm{Pd}$ catalysts, solvents, and bases were also examined. The catalysts and solvents, which provided poor to moderate yields, are listed in Table 1 (Table 1, entries 2-10). In contrast, the palladium catalyst, $\mathrm{PdCl}_{2}\left(\mathrm{PPh}_{3}\right)_{2}$, is the only catalyst, which afforded the highest yield $(80 \%)$ of the desired product $4 \mathbf{a}$ in the pres- ence of $\mathrm{K}_{2} \mathrm{CO}_{3}$ in DMF within 2.5 hours (Table 1, entry 11). When $\mathrm{K}_{2} \mathrm{CO}_{3}$ was replaced with an organic base such as $\mathrm{Et}_{3} \mathrm{~N}$, a lower yield (60\%) of the product $\mathbf{4 a}$ was noticed within 10 hours in the presence of the same catalyst and solvent (Table 1, entry 12). The same catalyst with $\mathrm{K}_{2} \mathrm{CO}_{3}$ in other solvents like DMSO and 1,4-dioxane gave moderate yields of the product $4 \mathbf{a}$ (Table 1 , entries 13,14 ). The reaction did not proceed in the absence of $\mathrm{Pd}$ catalyst (Table 1 , entry 15$)$.

With the help of optimized reaction conditions, the further scope and generality of this process for pyrimidine formation and Sonogashira coupling were examined and the results obtained are presented in Table 2.

Encouraged by these results, the possibilities of the construction of pyrimidine scaffold and Heck coupling in a single step were examined. Initially, a reaction was carried out using enaminone 1, 3-bromobenzimidamide hydrochloride (2), and methyl acrylate (5a) in the presence of $\mathrm{PdCl}_{2}\left(\mathrm{PPh}_{3}\right)_{2}$ and $\mathrm{K}_{2} \mathrm{CO}_{3}$ in DMF at $80-85^{\circ} \mathrm{C}$. The reaction proceeded smoothly to give the corresponding product $\mathbf{6 a}$ in $83 \%$ yield. Other alkenes were examined

Table 1 Optimization of the Reaction Conditions for the Formation of 3-[3-(4-phenylpyrimidin-2-yl)phenyl]prop-2-yn-1-ol ${ }^{\mathrm{a}}$

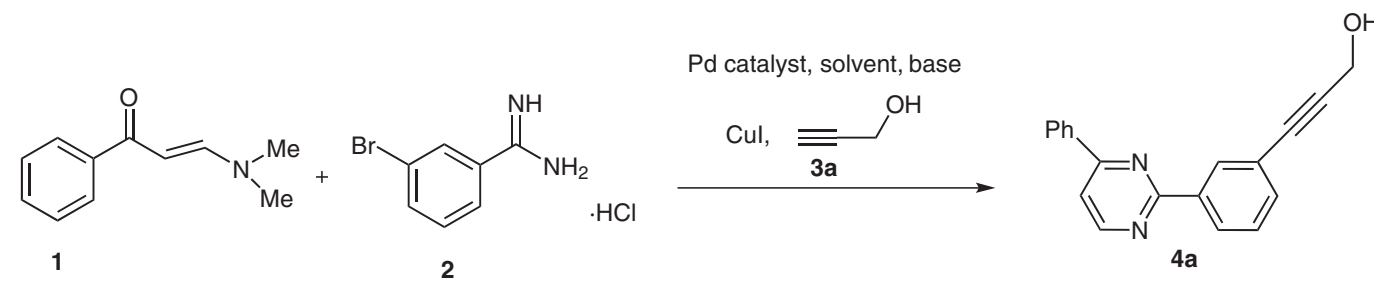

\begin{tabular}{|c|c|c|c|c|c|}
\hline Entry & Catalyst & Solvent & Base & Time (h) & Yield $(\%)^{b}$ \\
\hline 1 & $10 \% \mathrm{Pd} / \mathrm{C}$ & DMF & $\mathrm{K}_{2} \mathrm{CO}_{3}$ & 24 & 10 \\
\hline 2 & $20 \% \mathrm{Pd}(\mathrm{OH})_{2}$ & DMF & $\mathrm{K}_{2} \mathrm{CO}_{3}$ & 24 & 40 \\
\hline 3 & $20 \% \mathrm{Pd}(\mathrm{OH})_{2}$ & DMSO & $\mathrm{K}_{2} \mathrm{CO}_{3}$ & 24 & 30 \\
\hline 4 & $20 \% \mathrm{Pd}(\mathrm{OH})_{2}$ & 1,4-dioxane & $\mathrm{K}_{2} \mathrm{CO}_{3}$ & 24 & 25 \\
\hline 5 & $\mathrm{Pd}(\mathrm{dppf}) \mathrm{Cl}_{2}$ & DMF & $\mathrm{K}_{2} \mathrm{CO}_{3}$ & 14 & 55 \\
\hline 6 & $\mathrm{Pd}(\mathrm{dppf}) \mathrm{Cl}_{2}$ & DMSO & $\mathrm{K}_{2} \mathrm{CO}_{3}$ & 20 & 40 \\
\hline 7 & $\mathrm{Pd}(\mathrm{dppf}) \mathrm{Cl}_{2}$ & 1,4-dioxane & $\mathrm{K}_{2} \mathrm{CO}_{3}$ & 20 & 42 \\
\hline 8 & $\mathrm{Pd}\left(\mathrm{PPh}_{3}\right)_{4}$ & DMF & $\mathrm{K}_{2} \mathrm{CO}_{3}$ & 07 & 55 \\
\hline 9 & $\mathrm{Pd}\left(\mathrm{PPh}_{3}\right)_{4}$ & DMSO & $\mathrm{K}_{2} \mathrm{CO}_{3}$ & 10 & 50 \\
\hline 10 & $\mathrm{Pd}\left(\mathrm{PPh}_{3}\right)_{4}$ & 1,4-dioxane & $\mathrm{K}_{2} \mathrm{CO}_{3}$ & 10 & 45 \\
\hline 11 & $\mathrm{PdCl}_{2}\left(\mathrm{PPh}_{3}\right)_{2}$ & DMF & $\mathrm{K}_{2} \mathrm{CO}_{3}$ & 2.5 & 80 \\
\hline 12 & $\mathrm{PdCl}_{2}\left(\mathrm{PPh}_{3}\right)_{2}$ & DMF & $\mathrm{Et}_{3} \mathrm{~N}$ & 10 & 60 \\
\hline 13 & $\mathrm{PdCl}_{2}\left(\mathrm{PPh}_{3}\right)_{2}$ & DMSO & $\mathrm{K}_{2} \mathrm{CO}_{3}$ & 07 & 65 \\
\hline 14 & $\mathrm{PdCl}_{2}\left(\mathrm{PPh}_{3}\right)_{2}$ & 1,4-dioxane & $\mathrm{K}_{2} \mathrm{CO}_{3}$ & 07 & 60 \\
\hline 15 & - & DMF & $\mathrm{K}_{2} \mathrm{CO}_{3}$ & 24 & 0 \\
\hline
\end{tabular}

${ }^{\mathrm{a}}$ The reaction was carried out using $1(0.41 \mathrm{~g}, 2.339 \mathrm{mmol}), 2(0.5 \mathrm{~g}, 2.123 \mathrm{mmol})$, Pd catalyst $(0.0712 \mathrm{mmol})$, base $(1.02 \mathrm{~g}, 7.380 \mathrm{mmol}), \mathrm{CuI}$ $(0.04 \mathrm{~g}, 0.21 \mathrm{mmol})$, and prop-2-yn-1-ol $(0.128 \mathrm{~g}, 2.282 \mathrm{mmol})$ in a solvent $(2.5 \mathrm{~mL})$ at $70-75^{\circ} \mathrm{C}$.

${ }^{\mathrm{b}}$ Isolated yield. 
Table 2 Synthesis of 4-Phenyl-2-[3-(alkynyl)phenyl]pyrimidines 4 via Sonogashira Coupling MCR ${ }^{a}$<smiles>CN(C)/C=C/C(=O)c1ccccc1</smiles>

1

$$
\equiv{ }^{\mathrm{OH}}
$$

$3 a$

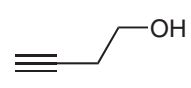

3b<smiles>C#CC(C)(C)O</smiles>

$3 \mathbf{c}$<smiles>C#CCCCO</smiles>

3d

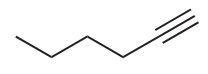

3 e

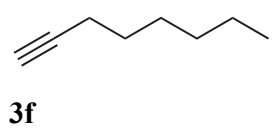

$\mathrm{DMF}, \mathrm{K}_{2} \mathrm{CO}_{3}$

$\mathrm{PdCl}_{2}\left(\mathrm{PPh}_{3}\right)_{2}, \mathrm{Cul}, \mathrm{R}-$

3

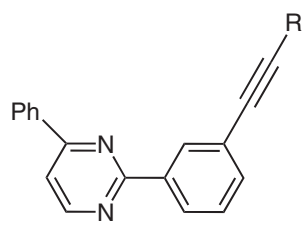

4

Time (h)

Yield (\%) $)_{f}^{\mathrm{b}}$

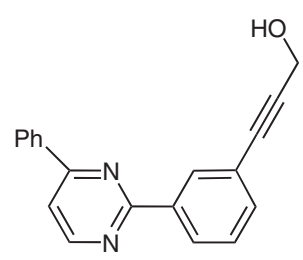

2.5

80

0.3

4a

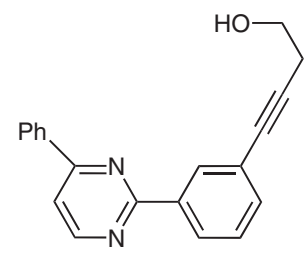

3.0

78

0.2

4b

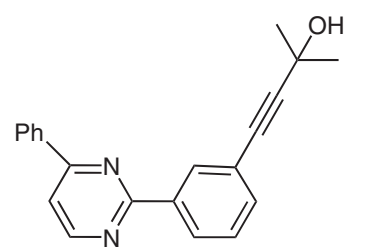

4.0

70

0.22

$4 c$<smiles>OCCCC#Cc1cccc(-c2nccc(-c3ccccc3)n2)c1</smiles>

4d

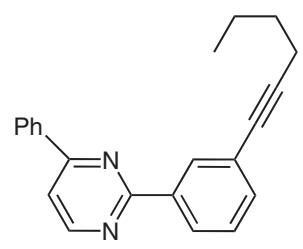

$4 e$

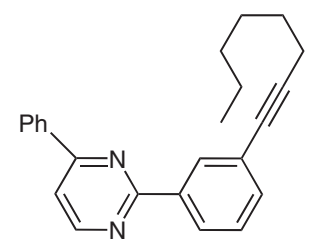

4f

${ }^{a}$ All the reactions were carried out using $\mathbf{1}(2.339 \mathrm{mmol}), \mathbf{2}(2.123 \mathrm{mmol}), \mathrm{CuI}(0.21 \mathrm{mmol})$, terminal alkyne derivatives 3a-f $(2.282 \mathrm{mmol})$, $\operatorname{DMF}(2.5 \mathrm{~mL}), \mathrm{PdCl}_{2}\left(\mathrm{PPh}_{3}\right)_{2}(50 \mathrm{mg}, 0.0712 \mathrm{mmol}), \mathrm{K}_{2} \mathrm{CO}_{3}(7.380 \mathrm{mmol})$ at $70-75^{\circ} \mathrm{C}$.

${ }^{\mathrm{b}}$ Isolated yield.

${ }^{\mathrm{c}}$ Retention factor; eluent: $10 \%$ EtOAc in PE. 
and the results obtained are presented in Table 3 . All reactions required 5-7 hours for completion and to provide the desired alkene derivatives 6a-f. Based on ${ }^{1} \mathrm{H}$ NMR data all the prepared alkenes were confirmed as $E$-isomers $(J=16.8-16.0 \mathrm{~Hz})$.

Table 3 Synthesis of (E)-4-Phenyl-2-[3-(alkenyl)phenyl]pyrimidines 6 via Heck Coupling MCR ${ }^{\mathrm{a}}$<smiles>CN(C)C=CC(=O)c1ccccc1</smiles>

1<smiles>N=C(N)c1cccc(Br)c1</smiles>

2

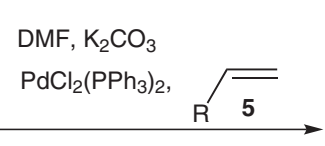

5.0

83

0.2

$5 \mathbf{a}$<smiles>COC(=O)/C=C/c1cccc(-c2nccc(-c3ccccc3)n2)c1</smiles>

Time (h)

Yield (\%) $\quad R_{f}^{\mathrm{c}}$
$6 a$<smiles>C=CC(=O)OCC</smiles>

$5 \mathbf{b}$<smiles>CCOC(=O)/C=C/c1cccc(-c2nccc(-c3ccccc3)n2)c1</smiles>

6b<smiles>C=CC(=O)OCCCC</smiles>

$5 \mathbf{c}$<smiles>CC(C)(C)OC(=O)/C=C/c1cccc(-c2nccc(-c3ccccc3)n2)c1</smiles>

$6 c$<smiles>C=CC(C)=O</smiles>

$5 \mathbf{d}$<smiles>CC(=O)/C=C/c1cccc(-c2nccc(-c3ccccc3)n2)c1</smiles>

6d<smiles>N#C/C=C/c1cccc(-c2nccc(-c3ccccc3)n2)c1</smiles>

$6 e$<smiles>C=CC(N)=O</smiles>

$5 f$

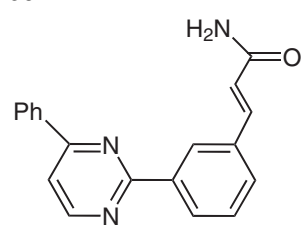

$6 f$

${ }^{a}$ All the reactions were carried out using 1 (2.339 mmol), 2 (2.123 mmol), $\mathrm{PdCl}_{2}\left(\mathrm{PPh}_{3}\right)_{2}(50 \mathrm{mg}, 0.0712 \mathrm{mmol})$, alkene derivatives $5 \mathbf{a}-\mathbf{f}(2.555$ $\mathrm{mmol}), \mathrm{K}_{2} \mathrm{CO}_{3}(7.380 \mathrm{mmol})$ in $\mathrm{DMF}(2.5 \mathrm{~mL})$ at $80-85^{\circ} \mathrm{C}$.

${ }^{\mathrm{b}}$ Isolated yield.

${ }^{\mathrm{c}}$ Retention factor; eluent: 15\% EtOAc in PE. 
Table 4 Synthesis of 2-(Biphenyl-3-yl)-4-phenylpyrimidines 8 via Suzuki Coupling MCR<smiles>C/C=C(/C)Br</smiles>

$7 \mathbf{a}$

2<smiles>COc1ccc([Se]O)cc1</smiles>

$7 \mathbf{b}$

3<smiles>COc1cccc([18OH])c1</smiles>

$7 c$

4<smiles>OCc1cccc(Br)c1</smiles>

$7 \mathbf{d}$<smiles></smiles>

$7 e$

6

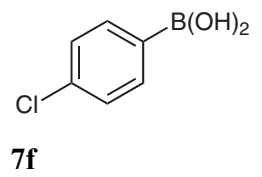

DMF, $\mathrm{K}_{2} \mathrm{CO}_{3}$

$\mathrm{PdCl}_{2}\left(\mathrm{PPh}_{3}\right)_{2}, \mathrm{ArB}(\mathrm{OH})_{2} 7$<smiles>Brc1cccc(-c2nccc(-c3ccccc3)n2)c1</smiles>

Yield (\%) ${ }^{\mathrm{b}} \quad R_{f}^{\mathrm{c}}$<smiles>N#Cc1cc(F)cc(-c2cccc(-c3nccc(-c4ccccc4)n3)c2)c1</smiles>

2.0

90

0.3

$8 a$<smiles>COc1ccc(-c2cccc(-c3nccc(-c4ccccc4)n3)c2)cc1</smiles>

2.6

85

0.4

$8 b$<smiles>COc1cccc(-c2cccc(-c3nccc(-c4ccccc4)n3)c2)c1</smiles>

80

0.4

$8 c$<smiles>OCc1cccc(-c2cccc(-c3nccc(-c4ccccc4)n3)c2)c1</smiles>

3.5

74

0.2

8d<smiles>FC(F)(F)c1ccc(-c2cccc(-c3nccc(-c4ccccc4)n3)c2)cc1</smiles>

2.0

85

0.4

$8 \mathbf{e}$

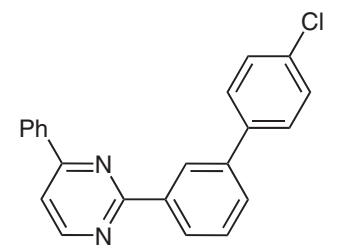

3.2

75

0.45

$8 f$ 
Table 4 Synthesis of 2-(Biphenyl-3-yl)-4-phenylpyrimidines 8 via Suzuki Coupling MCR ${ }^{\mathrm{a}}$ (continued)

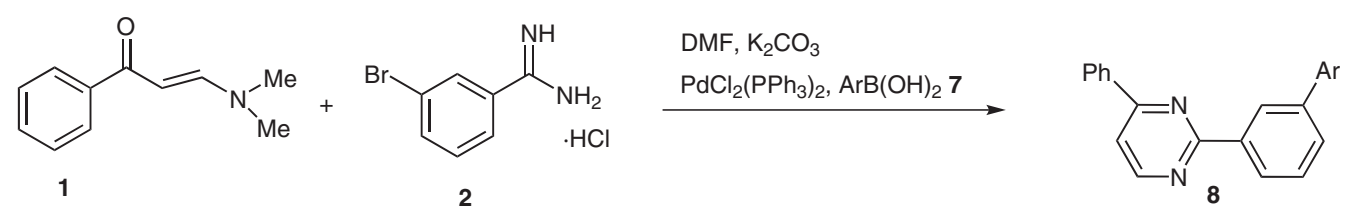

\begin{tabular}{llllll}
\hline Entry & Arylboronic acid & Product & Time (h) & Yield $(\%)^{\mathrm{b}}$ & $R_{f}^{\mathrm{c}}$ \\
\hline
\end{tabular}

7<smiles>COc1ccc(OC)nc1</smiles>

$7 \mathrm{~g}$

8<smiles>Oc1ccc(Cl)c(Cl)c1</smiles>

$7 \mathbf{h}$

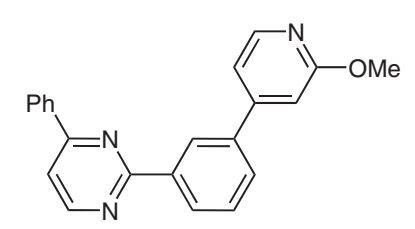

$8 \mathrm{~g}$

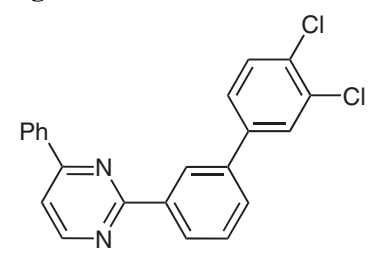

3.5

78

0.4
The possibilities for the formation of pyrimidine ring followed by Suzuki coupling in a single step were also examined. Initially, the reaction was carried out using enaminone 1, 3-bromobenzimidamide hydrochloride (2), and 3-cyano-5-fluorophenylboronic acid (7a) in the presence of $\mathrm{PdCl}_{2}\left(\mathrm{PPh}_{3}\right)_{2}$ and $\mathrm{K}_{2} \mathrm{CO}_{3}$ in $\mathrm{DMF}$ at $80-85{ }^{\circ} \mathrm{C}$. The reaction proceeded smoothly and afforded the corresponding product $8 \mathbf{a}$ in $90 \%$ yield. Different types of boronic acids were examined and the obtained results are summarized in Table 4 . All the reactions were completed within 2-3.5 hours and afforded the desired products $\mathbf{8 a}-\mathbf{h}$.

The plausible mechanism of MCR may proceed via a base-catalyzed Michael addition of 3-bromobenzimidamide hydrochloride (2) to the enaminone 1 to form the intermediate 9. This intermediate sequentially undergoes cyclization, isomerization, and dehydration to give 2-(3bromophenyl)-4-phenylpyrimidine (10), followed by Sonogashira, Heck or Suzuki coupling to afford the 4phenyl-2-(3-alkynyl/alkenyl/aryl)phenyl-substituted pyrimidines $\mathbf{4 , 6}$, and 8 as shown in Scheme 2 .

In conclusion, we have developed a new MCR strategy for the synthesis of 4-phenyl-2-[3-(alkynyl/alkenyl/aryl)phenyl]-substituted pyrimidines in high yields from enaminone 1, 3-bromobenzimidamide hydrochloride (2), and various alkynes/alkenes/arylboronic acids via a Michael addition, cyclization, isomerization, dehydration, and followed by Sonogashira/Heck/Suzuki coupling in a single pot. This MCR strategy offers several advantages like short reaction time, easy isolation of products, simple construction of substituted pyrimidine moiety, and subsequent $\mathrm{C}-\mathrm{C}$ couplings for the structural elaboration of pyrimidine framework in one pot.

Melting points were determined using a melting point apparatus and are uncorrected. ${ }^{1} \mathrm{H}$ NMR and ${ }^{13} \mathrm{C}$ NMR spectra were recorded on a Varian $400 \mathrm{MHz}$ spectrometer. Chemical shifts are expressed in parts per million (ppm) and coupling constants in hertz (Hz). Standard abbreviations were used to describe the splitting pattern. Highresolution mass spectra (HRMS) were recorded on a Waters LCT Premier XE mass spectrometer equipped with electrospray ionization (ESI) source. TLC was performed on $0.25 \mathrm{~mm}$ Merck silica gel plates and visualized with UV light. Column chromatography was performed on silica gel. Enaminone $\mathbf{1}$ and 3-bromobenzimidamide hydrochloride (2) were prepared according to the known procedure $^{23,24}$ and other chemicals and solvents were purchased from Sigma Aldrich and Merck and used directly. Petroleum ether (PE) used refers to the fraction boiling at $60-80^{\circ} \mathrm{C}$.

\section{3-[3-(4-Phenylpyrimidin-2-yl)phenyl]prop-2-yn-1-ol (4a); Typ-} ical Procedure

In a $25 \mathrm{~mL}$ round-bottomed flask were charged enaminone $1(0.41$ g, $2.339 \mathrm{mmol})$, 3-bromobenzimidamide hydrochloride $(2 ; 0.5 \mathrm{~g}$, $2.123 \mathrm{mmol})$, DMF $(2.5 \mathrm{~mL}), \mathrm{K}_{2} \mathrm{CO}_{3}(1.02 \mathrm{~g}, 7.380 \mathrm{mmol})$, $\mathrm{PdCl}_{2}\left(\mathrm{PPh}_{3}\right)_{2}(50 \mathrm{mg}, 0.0712 \mathrm{mmol})$, $\mathrm{CuI}(0.04 \mathrm{~g}, 0.21 \mathrm{mmol})$ and prop-1-yn-3-ol (3a; $0.128 \mathrm{~g}, 2.282 \mathrm{mmol})$ at r.t. Then, the reaction mixture was stirred at $70-75^{\circ} \mathrm{C}$ for $2.5 \mathrm{~h}$ and the reaction was monitored by TLC (10\% EtOAc in PE). After completion of the reaction, the mixture was concentrated under vacuum and the obtained crude product $4 \mathbf{a}$ was purified by column chromatography on silica gel (230-400 mesh) using EtOAc-PE (10\% EtOAc-PE); yield: 486 mg (80\%); off-white solid; mp $95.1-97.4{ }^{\circ} \mathrm{C}$. 


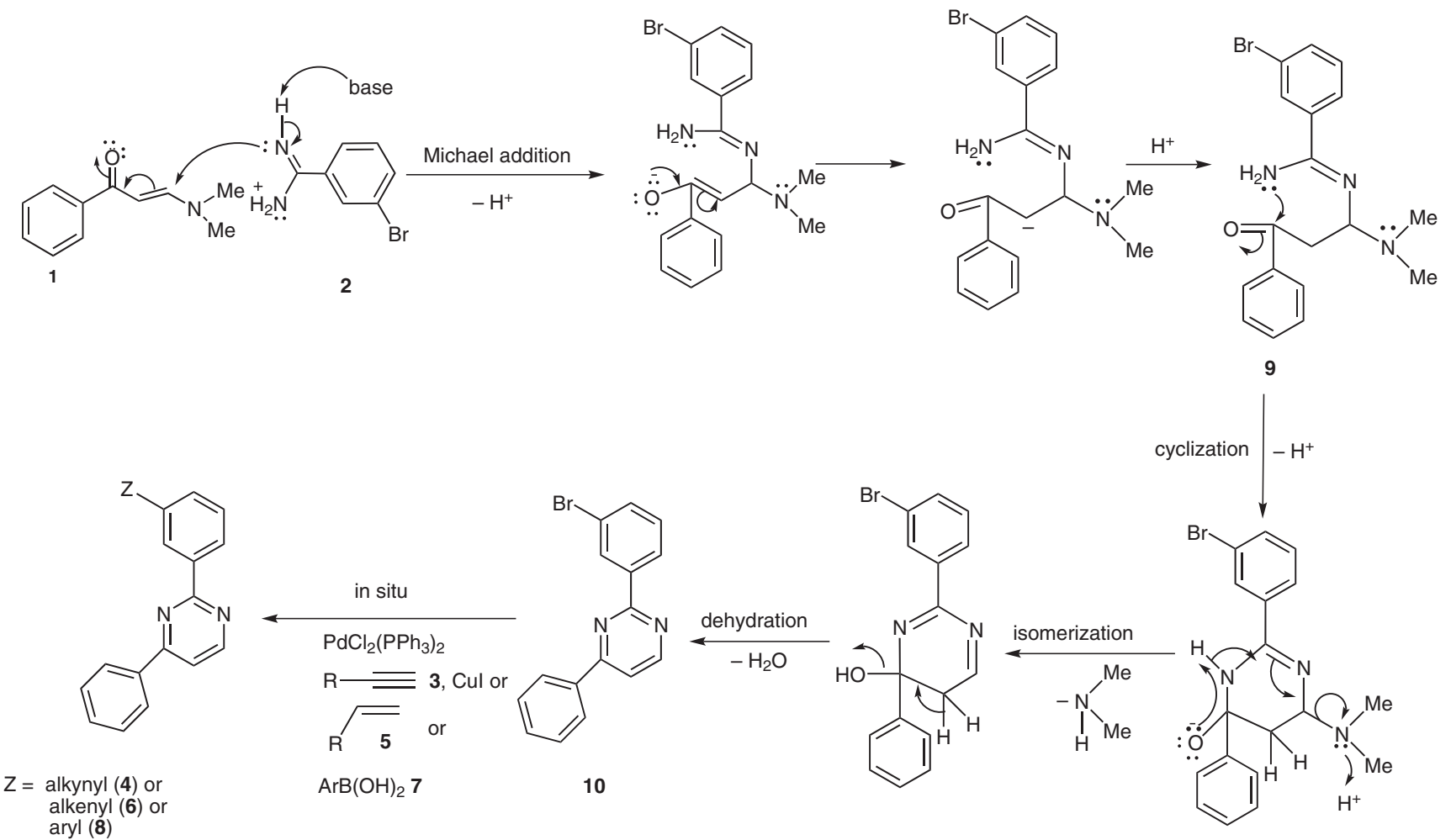

Scheme 2 Plausible mechanism for the formation of 4-phenyl-2-[3-(alkynyl/alkenyl/aryl)phenyl]-substituted pyrimidines

${ }^{1} \mathrm{H}$ NMR $\left(400 \mathrm{MHz}, \mathrm{CDCl}_{3}\right): \delta=8.83\left(\mathrm{~d}, J=4.8 \mathrm{~Hz}, 1 \mathrm{H}_{\text {arom }}\right), 8.55$ $\left(\mathrm{d}, J=8.4 \mathrm{~Hz}, 2 \mathrm{H}_{\text {arom }}\right), 8.21-8.20\left(\mathrm{~m}, 2 \mathrm{H}_{\text {arom }}\right), 7.60-7.25(\mathrm{~m}, 6$ $\left.\mathrm{H}_{\text {arom }}\right), 4.53\left(\mathrm{~s}, 2 \mathrm{H}, \mathrm{OCH}_{2}\right), 1.97(\mathrm{br}, 1 \mathrm{H}, \mathrm{OH})$.

${ }^{13} \mathrm{C} \mathrm{NMR}\left(400 \mathrm{MHz}, \mathrm{CDCl}_{3}\right): \delta=163.9,163.7,157.7,137.6,136.6$, 131.8 (2 C), 131.0, 128.9 (2 C), 128.1 (2 C), 127.1 (2 C), 124.8 114.6, 89.1, 85.5, 51.5 .

HRMS (ESI): $m / z(\mathrm{M}+\mathrm{H})^{+}$calcd for $\mathrm{C}_{19} \mathrm{H}_{15} \mathrm{~N}_{2} \mathrm{O}: 287.1184$; found: 287.1173 .

\section{4-[3-(4-Phenylpyrimidin-2-yl)phenyl]but-3-yn-1-ol (4b)}

Purification by column chromatography (15\% EtOAc-PE) gave $4 \mathbf{b}$ as an off-white solid; yield: $497 \mathrm{mg}(78 \%) ; \mathrm{mp} 94.2-96.3^{\circ} \mathrm{C}$.

${ }^{1} \mathrm{H}$ NMR (400 MHz, $\left.\mathrm{CDCl}_{3}\right): \delta=8.83\left(\mathrm{~d}, J=5.6 \mathrm{~Hz}, 1 \mathrm{H}_{\text {arom }}\right), 8.53$ $\left(\mathrm{d}, J=8.4 \mathrm{~Hz}, 2 \mathrm{H}_{\text {arom }}\right), 8.23-8.20\left(\mathrm{~m}, 2 \mathrm{H}_{\text {arom }}\right), 7.60(\mathrm{~d}, J=5.2 \mathrm{~Hz}$, $\left.1 \mathrm{H}_{\text {arom }}\right), 7.55-7.53\left(\mathrm{~m}, 5 \mathrm{H}_{\text {arom }}\right), 3.86\left(\mathrm{t}, J=6.4 \mathrm{~Hz}, 2 \mathrm{H}, \mathrm{OCH}_{2}\right)$, $2.60\left(\mathrm{t}, J=6.8 \mathrm{~Hz}, 2 \mathrm{H}, \mathrm{CH}_{2}\right)$.

${ }^{13} \mathrm{C} \mathrm{NMR}\left(400 \mathrm{MHz}, \mathrm{CDCl}_{3}\right): \delta=163.9,163.8,157.7,136.9,136.8$, 131.7 (2 C), 130.9, 128.9 (2 C), 128.0 (2 C), 127.1 (2 C), 126.0, 114.5, 91.4, 81.7, 61.7, 16.0.

HRMS (ESI): $m / z(\mathrm{M}+\mathrm{H})^{+}$calcd for $\mathrm{C}_{20} \mathrm{H}_{17} \mathrm{~N}_{2} \mathrm{O}: 301.1341$; found: 301.1347 .

\section{2-Methyl-4-[3-(5-phenylpyrimidin-2-yl)phenyl]but-3-yn-2-ol}

\section{(4c)}

Purification by column chromatography (20\% EtOAc-PE) gave $4 \mathbf{c}$ as an off-white solid; yield: $467 \mathrm{mg}(70 \%)$; mp 95.3-97.7 ${ }^{\circ} \mathrm{C}$.

${ }^{1} \mathrm{H}$ NMR $\left(400 \mathrm{MHz}, \mathrm{CDCl}_{3}\right): \delta=8.84\left(\mathrm{~d}, J=5.6 \mathrm{~Hz}, 1 \mathrm{H}_{\text {arom }}\right), 8.54$ $\left(\mathrm{d}, J=8.8 \mathrm{~Hz}, 2 \mathrm{H}_{\text {arom }}\right), 8.23-8.21\left(\mathrm{~m}, 2 \mathrm{H}_{\text {arom }}\right), 7.61(\mathrm{~d}, J=4.8 \mathrm{~Hz}$, $\left.1 \mathrm{H}_{\text {arom }}\right), 7.62-7.54\left(\mathrm{~m}, 5 \mathrm{H}_{\text {arom }}\right), 1.50\left[6 \mathrm{H}, \mathrm{C}\left(\mathrm{CH}_{3}\right)_{2}\right]$.

${ }^{13} \mathrm{C} \mathrm{NMR}\left(400 \mathrm{MHz}, \mathrm{CDCl}_{3}\right): \delta=163.9,163.8,157.8,137.4,136.7$, 131.8 (2 C), 131.0, 128.9 (2 C), 128.1 (2 C), 127.2 (2 C), 125.0, $114.6,83.9,66.3,31.4,31.0(2 \mathrm{C})$.
HRMS (ESI): $m / z(\mathrm{M}+\mathrm{H})^{+}$calcd for $\mathrm{C}_{21} \mathrm{H}_{19} \mathrm{~N}_{2} \mathrm{O}: 315.1497$; found: 315.1486 .

5-[3-(4-Phenylpyrimidin-2-yl)phenyl]pent-4-yn-1-ol (4d)

Purification by column chromatography (15\% EtOAc-PE) gave 4d as an off-white solid; yield: $533 \mathrm{mg}(80 \%)$; mp 94.3-96.6 ${ }^{\circ} \mathrm{C}$.

${ }^{1} \mathrm{H} \mathrm{NMR}\left(400 \mathrm{MHz}, \mathrm{CDCl}_{3}\right): \delta=8.83\left(\mathrm{~d}, J=5.6 \mathrm{~Hz}, 1 \mathrm{H}_{\text {arom }}\right), 8.52$ $\left(\mathrm{d}, J=8.4 \mathrm{~Hz}, 2 \mathrm{H}_{\text {arom }} \mathrm{H}\right), 8.23-8.20\left(\mathrm{~m}, 2 \mathrm{H}_{\text {arom }}\right), 7.60(\mathrm{~d}, J=5.2$ $\left.\mathrm{Hz}, 1 \mathrm{H}_{\text {arom }}\right), 7.55-7.52\left(\mathrm{~m}, 5 \mathrm{H}_{\text {arom }}\right), 3.85\left(\mathrm{t}, J=6.4 \mathrm{~Hz}, 2 \mathrm{H}, \mathrm{OCH}_{2}\right)$, $2.61\left(\mathrm{t}, J=6.8 \mathrm{~Hz}, 2 \mathrm{H}, \mathrm{CH}_{2}\right), 1.90$ (quint, $J=6.4 \mathrm{~Hz}, 2 \mathrm{H}, \mathrm{CH}_{2}$ ).

${ }^{13} \mathrm{C} \mathrm{NMR}\left(400 \mathrm{MHz}, \mathrm{CDCl}_{3}\right): \delta=163.8,157.8,137.7,136.7,131.8$ (2 C), 131.0, $128.9(2 \mathrm{C}), 128.1(2 \mathrm{C}), 127.2(2 \mathrm{C}), 124.8,114.6$, $85.6,51.6,31.9,14.1$.

HRMS (ESI): $m / z(\mathrm{M}+\mathrm{H})^{+}$calcd for $\mathrm{C}_{21} \mathrm{H}_{19} \mathrm{~N}_{2} \mathrm{O}: 315.1497$; found: 315.1482 .

\section{2-[3-(Hex-1-ynyl)phenyl]-4-phenylpyrimidine (4e)}

Purification by column chromatography $(10 \%$ EtOAc-PE) gave $4 \mathrm{e}$ as an off-white solid; yield: $550 \mathrm{mg}(83 \%)$; mp $109.4-111.5^{\circ} \mathrm{C}$.

${ }^{1} \mathrm{H} \mathrm{NMR}\left(400 \mathrm{MHz}, \mathrm{CDCl}_{3}\right): \delta=8.82\left(\mathrm{~d}, J=5.6 \mathrm{~Hz}, 1 \mathrm{H}_{\text {arom }}\right), 8.51$ $\left(\mathrm{d}, J=8.4 \mathrm{~Hz}, 2 \mathrm{H}_{\text {arom }}\right), 8.23-8.20\left(\mathrm{~m}, 2 \mathrm{H}_{\text {arom }}\right), 7.59(\mathrm{~d}, J=5.2 \mathrm{~Hz}$, $\left.1 \mathrm{H}_{\text {arom }}\right), 7.55-7.52\left(\mathrm{~m}, 5 \mathrm{H}_{\text {arom }}\right), 2.46\left(\mathrm{t}, J=6.8 \mathrm{~Hz}, 2 \mathrm{H}, \mathrm{CH}_{2}\right)$, 1.64-1.48 (m, $\left.4 \mathrm{H}, 2 \mathrm{CH}_{2}\right), 0.98$ (t, $\left.J=7.2 \mathrm{~Hz}, 3 \mathrm{H}, \mathrm{CH}_{3}\right)$.

${ }^{13} \mathrm{C} \mathrm{NMR}\left(400 \mathrm{MHz}, \mathrm{CDCl}_{3}\right): \delta=162.9,162.8,156.7,135.8,135.7$, 130.6 (2 C), 129.9, 127.8 (2 C), 127.0 (2 C), 126.1 (2 C), 125.4, $113.4,91.5,79.6,29.7,21.0,18.2,12.6$.

HRMS (ESI): $m / z(\mathrm{M}+\mathrm{H})^{+}$calcd for $\mathrm{C}_{22} \mathrm{H}_{21} \mathrm{~N}_{2}: 313.1705$; found: 313.1693 .

\section{2-[3-(Oct-1-ynyl)phenyl]-4-phenylpyrimidine (4f)}

Purification by column chromatography (10\% EtOAc-PE) gave $\mathbf{4 f}$ as an off-white solid; yield: $614 \mathrm{mg}(85 \%)$; mp 113.2-115.4 ${ }^{\circ} \mathrm{C}$. 
${ }^{1} \mathrm{H} \mathrm{NMR}\left(400 \mathrm{MHz}, \mathrm{CDCl}_{3}\right): \delta=8.82\left(\mathrm{~d}, J=5.6 \mathrm{~Hz}, 1 \mathrm{H}_{\text {arom }}\right), 8.51$ $\left(\mathrm{d}, J=8.4 \mathrm{~Hz}, 2 \mathrm{H}_{\text {arom }}\right), 8.23-8.20\left(\mathrm{~m}, 2 \mathrm{H}_{\text {arom }}\right), 7.59(\mathrm{~d}, J=5.6 \mathrm{~Hz}$, $\left.1 \mathrm{H}_{\text {arom }}\right), 7.54-7.52\left(\mathrm{~m}, 5 \mathrm{H}_{\text {arom }}\right), 2.45\left(\mathrm{t}, J=7.2 \mathrm{~Hz}, 2 \mathrm{H}, \mathrm{CH}_{2}\right)$, 1.67-1.44 (m, $\left.4 \mathrm{H}, 2 \mathrm{CH}_{2}\right), 1.35-1.33\left(\mathrm{~m}, 4 \mathrm{H}, 2 \mathrm{CH}_{2}\right), 0.93(\mathrm{t}$, $\left.J=6.8 \mathrm{~Hz}, 3 \mathrm{H}, \mathrm{CH}_{3}\right)$.

${ }^{13} \mathrm{C} \mathrm{NMR}\left(400 \mathrm{MHz}, \mathrm{CDCl}_{3}\right): \delta=164.0,163.8,157.7,136.8,136.7$, 131.6 (2 C), 130.9, 128.8 (2 C), 128.0 (2 C), 127.1 (2 C), 126.4, 114.4, 92.6, 80.6, 31.3, 28.67, 28.63, 22.5, 19.5, 14.0

HRMS (ESI): $m / z(\mathrm{M}+\mathrm{H})^{+}$calcd for $\mathrm{C}_{24} \mathrm{H}_{25} \mathrm{~N}_{2}: 341.2018$; found: 341.2025 .

Methyl (E)-3-[3-(4-Phenylpyrimidin-2-yl)phenyl]acrylate (6a); Typical Procedure

In a $25 \mathrm{~mL}$ round-bottomed flask were charged enaminone $\mathbf{1}(0.41$ $\mathrm{g}, 2.339 \mathrm{mmol})$, 3-bromobenzimidamide hydrochloride $(2 ; 0.5 \mathrm{~g}$, $2.123 \mathrm{mmol})$, DMF $(2.5 \mathrm{~mL}), \mathrm{K}_{2} \mathrm{CO}_{3}(1.02 \mathrm{~g}, 7.380 \mathrm{mmol})$, $\mathrm{PdCl}_{2}\left(\mathrm{PPh}_{3}\right)_{2}(50 \mathrm{mg}, 0.0712 \mathrm{mmol})$, and methyl acrylate $(\mathbf{5 a} ; 0.22$ $\mathrm{g}, 2.555 \mathrm{mmol})$ at r.t. Then, the mixture was stirred at $80-85^{\circ} \mathrm{C}$ for $4-5 \mathrm{~h}$ and the reaction was monitored by TLC (10\% EtOAc in PE). After completion of the reaction, the mixture was concentrated under vacuum and the obtained crude product $\mathbf{6 a}$ was purified by column chromatography on silica gel (230-400 mesh) using 15\% EtOAc-PE; yield: $557 \mathrm{mg}$ (83\%); off-white solid; mp 153.2$155.5^{\circ} \mathrm{C}$.

${ }^{1} \mathrm{H}$ NMR $\left(400 \mathrm{MHz}, \mathrm{CDCl}_{3}\right): \delta=8.85\left(\mathrm{~d}, J=5.2 \mathrm{~Hz}, 1 \mathrm{H}_{\text {arom }}\right), 8.61$ $\left(\mathrm{d}, J=8.4 \mathrm{~Hz}, 2 \mathrm{H}_{\text {arom }}\right), 8.23-8.21\left(\mathrm{~m}, 2 \mathrm{H}_{\text {arom }}\right), 7.79(\mathrm{~d}, J=16.4 \mathrm{~Hz}$ $1 \mathrm{H}$, trans $\mathrm{H}), 7.68-7.54\left(\mathrm{~m}, 6 \mathrm{H}_{\text {arom }}\right), 6.56(\mathrm{~d}, J=16.0 \mathrm{~Hz}, 1 \mathrm{H}$, trans $\mathrm{H}), 3.83\left(\mathrm{~s}, \mathrm{OCH}_{3}\right)$.

${ }^{13} \mathrm{C} \mathrm{NMR}\left(400 \mathrm{MHz}, \mathrm{CDCl}_{3}\right): \delta=167.2,163.8,163.6,157.7,144.2$, 139.4, 136.6, 136.3, 131.0, 128.8 (2 C), 128.6 (2 C), 128.1 (2 C), 127.1 (2 C), 118.5, 114.6, 51.6.

HRMS (ESI): $m / z(\mathrm{M}+\mathrm{H})^{+}$calcd for $\mathrm{C}_{20} \mathrm{H}_{17} \mathrm{~N}_{2} \mathrm{O}_{2}: 317.1290$; found: 317.1287 .

\section{Ethyl (E)-3-[3-(4-Phenylpyrimidin-2-yl)phenyl]acrylate (6b)} Purification by column chromatography (15\% EtOAc-PE) gave $6 \mathbf{b}$ as an off-white solid; yield: $546 \mathrm{mg}(78 \%)$; mp $106.5-108.7^{\circ} \mathrm{C}$.

${ }^{1} \mathrm{H}$ NMR $\left(400 \mathrm{MHz}, \mathrm{CDCl}_{3}\right): \delta=8.84\left(\mathrm{~d}, J=5.2 \mathrm{~Hz}, 1 \mathrm{H}_{\text {arom }}\right), 8.60$ $\left(\mathrm{d}, J=8.4 \mathrm{~Hz}, 2 \mathrm{H}_{\text {arom }}\right), 8.23-8.20\left(\mathrm{~m}, 2 \mathrm{H}_{\text {arom }}\right), 7.76(\mathrm{~d}, J=16.4 \mathrm{~Hz}$, $1 \mathrm{H}$, trans $\mathrm{H}), 7.67\left(\mathrm{~d}, J=8.4 \mathrm{~Hz}, 2 \mathrm{H}_{\text {arom }}\right), 7.62(\mathrm{~d}, J=4.8 \mathrm{~Hz}, 1$ $\left.\mathrm{H}_{\text {arom }}\right), 7.56-7.53\left(\mathrm{~m}, 3 \mathrm{H}_{\text {arom }}\right), 6.54(\mathrm{~d}, J=16.0 \mathrm{~Hz}, 1 \mathrm{H}$, trans $\mathrm{H})$, $4.29\left(\mathrm{q}, J=6.8 \mathrm{~Hz}, 2 \mathrm{H}, \mathrm{OCH}_{2}\right), 1.36\left(\mathrm{t}, J=6.8 \mathrm{~Hz}, 3 \mathrm{H}, \mathrm{CH}_{3}\right)$.

${ }^{13} \mathrm{C} \mathrm{NMR}\left(400 \mathrm{MHz}, \mathrm{CDCl}_{3}\right): \delta=166.7,163.7,163.6,157.7,143.8$, 139.3, 136.6, 136.4, 130.9, 128.8 (2 C), 128.6 (2 C), 128.1 (2 C), 127.0 (2 C), 119.0, 114.6, 60.4, 14.2.

HRMS (ESI): $m / z(\mathrm{M}+\mathrm{H})^{+}$calcd for $\mathrm{C}_{21} \mathrm{H}_{19} \mathrm{~N}_{2} \mathrm{O}_{2}: 331.1447$; found: 331.1455 .

\section{tert-Butyl (E)-3-[3-(4-Phenylpyrimidin-2-yl)phenyl]acrylate (6c)}

Purification by column chromatography (15\% EtOAc-PE) gave $6 \mathbf{c}$ as an off-white solid; yield: $608 \mathrm{mg}(80 \%)$; $\mathrm{mp} 94.8-97.1^{\circ} \mathrm{C}$.

${ }^{1} \mathrm{H}$ NMR (400 MHz, $\left.\mathrm{CDCl}_{3}\right): \delta=8.84\left(\mathrm{~d}, J=5.2 \mathrm{~Hz}, 1 \mathrm{H}_{\text {arom }}\right), 8.59$ (d, $\left.J=8.8 \mathrm{~Hz}, 2 \mathrm{H}_{\text {arom }}\right), 8.24-8.20\left(\mathrm{~m}, 2 \mathrm{H}_{\text {arom }}\right), 7.68-7.53(\mathrm{~m}, 7$ $\mathrm{H}_{\text {arom }}$ and trans $\left.\mathrm{H}\right), 6.49(\mathrm{~d}, J=16.0 \mathrm{~Hz}, 1 \mathrm{H}$, trans $\mathrm{H}), 1.55(\mathrm{~s}, 9 \mathrm{H}$, $\left.t-\mathrm{C}_{4} \mathrm{H}_{9}\right)$.

${ }^{13} \mathrm{C} \mathrm{NMR}\left(400 \mathrm{MHz}, \mathrm{CDCl}_{3}\right): \delta=166.1,163.8,163.8,157.8(2 \mathrm{C})$, 142.9, 139.1, 136.7, 131.0, 128.9 (2 C), 128.6 (2 C), 128.0 (2 C), 127.1 (2 C), 121.0, 114.6, 80.5, 28.1 (3 C).

HRMS (ESI): $m / z(\mathrm{M}+\mathrm{H})^{+}$calcd for $\mathrm{C}_{23} \mathrm{H}_{23} \mathrm{~N}_{2} \mathrm{O}_{2}: 359.1760$; found: 359.1761 .

(E)-4-[3-(4-Phenylpyrimidin-2-yl)phenyl]but-3-en-2-one (6d) Purification by column chromatography $(10 \%$ EtOAc-PE) gave $6 \mathbf{d}$ as an off-white solid; yield: $497 \mathrm{mg}(78 \%)$; $\mathrm{mp} 173.1-175.5^{\circ} \mathrm{C}$.
${ }^{1} \mathrm{H}$ NMR $\left(400 \mathrm{MHz}, \mathrm{CDCl}_{3}\right): \delta=8.85\left(\mathrm{~d}, J=5.6 \mathrm{~Hz}, 1 \mathrm{H}_{\text {arom }}\right), 8.62$ $\left(\mathrm{d}, J=8.4 \mathrm{~Hz}, 2 \mathrm{H}_{\text {arom }}\right), 8.24-8.21\left(\mathrm{~m}, 2 \mathrm{H}_{\text {arom }}\right), 7.70(\mathrm{~d}, J=8.4 \mathrm{~Hz}$, $\left.1 \mathrm{H}_{\text {arom }}\right), 7.63-7.61\left(\mathrm{~m}, 1 \mathrm{H}_{\text {arom }}\right), 7.57-7.53\left(\mathrm{~m}, 3 \mathrm{H}_{\text {arom }}\right.$ and trans $\left.\mathrm{H}\right)$, $6.83(\mathrm{~d}, J=16.0 \mathrm{~Hz}, 1 \mathrm{H}$, trans $\mathrm{H}), 2.42\left(\mathrm{~s}, 3 \mathrm{H}, \mathrm{CH}_{3}\right)$.

${ }^{13} \mathrm{C} \mathrm{NMR}\left(400 \mathrm{MHz}, \mathrm{CDCl}_{3}\right): \delta=198.1,163.7,163.5,157.7,142.6$, 139.6, 136.5, 136.3, 131.0, 128.8 (2 C), 128.6 (2 C), 128.3 (2 C), 127.7, $127.0(2 \mathrm{C}), 114.7,27.4$.

HRMS (ESI): $m / z(\mathrm{M}+\mathrm{H})^{+}$calcd for $\mathrm{C}_{20} \mathrm{H}_{17} \mathrm{~N}_{2} \mathrm{O}: 301.1341$; found: 301.1349 .

(E)-3-[3-(4-Phenylpyrimidin-2-yl)phenyl]acrylonitrile (6e) Purification by column chromatography (20\% EtOAc-PE) gave $6 \mathbf{e}$ as an off-white solid; yield: $487 \mathrm{mg}(81 \%)$; $\mathrm{mp} 204.1-206.4{ }^{\circ} \mathrm{C}$.

${ }^{1} \mathrm{H} \mathrm{NMR}\left(400 \mathrm{MHz}, \mathrm{CDCl}_{3}\right): \delta=8.85\left(\mathrm{~d}, J=5.6 \mathrm{~Hz}, 1 \mathrm{H}_{\text {arom }}\right), 8.63$ $\left(\mathrm{d}, J=8.0 \mathrm{~Hz}, 2 \mathrm{H}_{\text {arom }}\right), 8.23-8.21\left(\mathrm{~m}, 2 \mathrm{H}_{\text {arom }}\right), 7.64-7.54(\mathrm{~m}, 6$ $\left.\mathrm{H}_{\text {arom }}\right), 7.48(\mathrm{~d}, J=16.4 \mathrm{~Hz}, 1 \mathrm{H}$, trans $\mathrm{H}), 6.00(\mathrm{~d}, J=16.8 \mathrm{~Hz}, 1$ $\mathrm{H}$, trans $\mathrm{H})$.

${ }^{13} \mathrm{C} \mathrm{NMR}\left(400 \mathrm{MHz}, \mathrm{CDCl}_{3}\right): \delta=166.5,162.9,162.7,158.6,138.4$, 138.0, 137.2, 136.1, 131.2, 129.0 (2 C), 128.3 (2 C), 127.9 (2 C), 127.1 (2 C), 123.5, 115.1 .

HRMS (ESI): $m / z(\mathrm{M}+\mathrm{H})^{+}$calcd for $\mathrm{C}_{19} \mathrm{H}_{14} \mathrm{~N}_{3}: 284.1188$; found: 284.1181 .

(E)-3-[3-(4-Phenylpyrimidin-2-yl)phenyl]acrylamide (6f)

Purification by column chromatography $(25 \%$ EtOAc-PE) gave 6 f as an off-white solid; yield: $467 \mathrm{mg}(73 \%)$; $\mathrm{mp} 195.3-197.6^{\circ} \mathrm{C}$.

${ }^{1} \mathrm{H} \mathrm{NMR}\left(400 \mathrm{MHz}, \mathrm{CDCl}_{3}\right): \delta=8.98\left(\mathrm{~d}, J=5.2 \mathrm{~Hz}, 1 \mathrm{H}_{\text {arom }}\right), 8.57$ $\left(\mathrm{d}, J=8.0 \mathrm{~Hz}, 2 \mathrm{H}_{\text {arom }}\right), 8.37-8.36\left(\mathrm{~m}, 2 \mathrm{H}_{\text {arom }}\right), 8.04(\mathrm{~d}, J=5.6 \mathrm{~Hz}$, $\left.1 \mathrm{H}_{\text {arom }}\right), 7.76\left(\mathrm{~d}, J=8.4 \mathrm{~Hz}, 2 \mathrm{H}_{\text {arom }}\right), 7.62-7.60\left(\mathrm{~m}, 4 \mathrm{H}_{\text {arom }}\right), 7.19$ (br, $1 \mathrm{H}, \mathrm{NH}), 7.52(\mathrm{~d}, J=16.4 \mathrm{~Hz}, 1 \mathrm{H}$, trans $\mathrm{H}), 6.76(\mathrm{~d}, J=16 \mathrm{~Hz}$, $1 \mathrm{H}$, trans $\mathrm{H})$.

${ }^{13} \mathrm{C} \mathrm{NMR}\left(400 \mathrm{MHz}, \mathrm{CDCl}_{3}\right): \delta=174.7,162.9,162.4,158.2,149.4$, $147.8,139.4,135.9,135.5,130.9,128.7$ (2 C), 128.1, 127.7, 126.8 (2 C), 118.1, 115.0, 97.7.

HRMS (ESI): $m / z(\mathrm{M}+\mathrm{H})^{+}$calcd for $\mathrm{C}_{19} \mathrm{H}_{16} \mathrm{~N}_{3} \mathrm{O}: 302.1293$; found: 302.1296 .

\section{5-Fluoro-3'-(4-phenylpyrimidin-2-yl)biphenyl-3-carbonitrile (8a); Typical Procedure}

In a $25 \mathrm{~mL}$ round-bottomed flask were charged enaminone $1(0.41$ g, $2.339 \mathrm{mmol})$, 3-bromobenzimidamide hydrochloride $(2 ; 0.5 \mathrm{~g}$, $2.123 \mathrm{mmol})$, DMF $(2.5 \mathrm{~mL}), \mathrm{K}_{2} \mathrm{CO}_{3}(1.02 \mathrm{~g}, 7.380 \mathrm{mmol})$, $\mathrm{PdCl}_{2}\left(\mathrm{PPh}_{3}\right)_{2}(50 \mathrm{mg}, 0.0712 \mathrm{mmol})$, and 3-cyano-5-fluorophenylboronic acid $(7 \mathbf{a} ; 0.42 \mathrm{~g}, 2.546 \mathrm{mmol})$ at r.t. The reaction mixture was then stirred at $80-85^{\circ} \mathrm{C}$ for $2 \mathrm{~h}$ and the reaction was monitored by TLC (15\% EtOAc in PE). After completion of the reaction, the mixture was concentrated under vacuum and the obtained crude product 8a was purified by column chromatography on silica gel (230-400 mesh) using 15\% EtOAc-PE; yield: $671 \mathrm{mg}$ (90\%); offwhite solid; $\mathrm{mp} 152.5-154.6^{\circ} \mathrm{C}$.

${ }^{1} \mathrm{H}$ NMR $\left(400 \mathrm{MHz}, \mathrm{CDCl}_{3}\right): \delta=8.87\left(\mathrm{~d}, J=5.2 \mathrm{~Hz}, 1 \mathrm{H}_{\mathrm{arom}}\right), 8.69$ $\left(\mathrm{d}, J=8.0 \mathrm{~Hz}, 2 \mathrm{H}_{\text {arom }}\right), 8.26-8.23\left(\mathrm{~m}, 2 \mathrm{H}_{\text {arom }}\right), 7.76\left(\mathrm{~s}, 1 \mathrm{H}_{\text {arom }}\right)$, $7.70\left(\mathrm{~d}, J=8.4 \mathrm{~Hz}, 2 \mathrm{H}_{\text {arom }}\right), 7.66\left(\mathrm{~d}, J=5.2 \mathrm{~Hz}, 2 \mathrm{H}_{\text {arom }}\right) 7.63-7.55$ (m, $\left.3 \mathrm{H}_{\text {arom }}\right), 7.37-7.35\left(\mathrm{~m}, 1 \mathrm{H}_{\text {arom }}\right)$.

${ }^{13} \mathrm{C}$ NMR $\left(400 \mathrm{MHz}, \mathrm{CDCl}_{3}\right): \delta=164.1,163.7(\mathrm{~d}, J=34.4 \mathrm{~Hz}, \mathrm{Ar}-$ CF), 161.2, 157.7 (2 C), 144.4, 139.5, 139.5, 138.3, 136.5, 131.1 (2 C), 129.1, 128.9, 127.1 (2 C), 127.1, 126.75, 126.71, 119.0, 118.8, 115.3, 114.8 .

HRMS (ESI): $m / z(\mathrm{M}+\mathrm{H})^{+}$calcd for $\mathrm{C}_{23} \mathrm{H}_{15} \mathrm{FN}_{3}: 352.1250$; found: 352.1255 .

2-(4'-Methoxybiphenyl-3-yl)-4-phenylpyrimidine (8b)

Purification by column chromatography (10\% EtOAc-PE) gave $\mathbf{8 b}$ as an off-white solid; yield: $610 \mathrm{mg}(85 \%)$; $\mathrm{mp} 159.2-161.5^{\circ} \mathrm{C}$.

$\left.{ }^{1} \mathrm{H} \mathrm{NMR} \mathrm{(400} \mathrm{MHz,} \mathrm{CDCl}_{3}\right): \delta=8.84\left(\mathrm{~d}, J=5.6 \mathrm{~Hz}, 1 \mathrm{H}_{\text {arom }}\right), 8.63$ $\left(\mathrm{d}, J=8.4 \mathrm{~Hz}, 2 \mathrm{H}_{\text {arom }}\right), 8.26-8.23\left(\mathrm{~m}, 2 \mathrm{H}_{\text {arom }}\right), 7.71(\mathrm{~d}, J=8.8 \mathrm{~Hz}$, 
$\left.2 \mathrm{H}_{\text {arom }}\right), 7.65-7.62\left(\mathrm{~m}, 2 \mathrm{H}_{\text {arom }}\right), 7.60\left(\mathrm{~d}, J=5.6 \mathrm{~Hz}, 2 \mathrm{H}_{\text {arom }}\right), 7.57-$ $7.54\left(\mathrm{~m}, 2 \mathrm{H}_{\text {arom }}\right), 7.02\left(\mathrm{~d}, J=8.8 \mathrm{~Hz} 2 \mathrm{H}_{\text {arom }}\right), 3.87\left(\mathrm{~s}, 3 \mathrm{H}, \mathrm{OCH}_{3}\right)$. ${ }^{13} \mathrm{C} \mathrm{NMR}\left(400 \mathrm{MHz}, \mathrm{CDCl}_{3}\right): \delta=164.3,163.8,159.4,157.7,142.9$, $136.8,136.0,132.9,130.9,128.8$ (2 C), 128.7 (2 C), 128.1 (2 C), 127.1 (2 C), 126.6 (2 C), 116.0, 114.2 (2 C), 55.2.

HRMS (ESI): $m / z(\mathrm{M}+\mathrm{H})^{+}$calcd for $\mathrm{C}_{23} \mathrm{H}_{19} \mathrm{~N}_{2} \mathrm{O}: 339.1497$; found: 339.1506 .

\section{2-(3'-Methoxybiphenyl-3-yl)-4-phenylpyrimidine (8c)}

Purification by column chromatography (10\% EtOAc-PE) gave $\mathbf{8 c}$ as an off-white solid; yield: $574 \mathrm{mg}(80 \%)$; $\mathrm{mp} 158.2-160.5^{\circ} \mathrm{C}$.

${ }^{1} \mathrm{H}$ NMR $\left(400 \mathrm{MHz}, \mathrm{CDCl}_{3}\right): \delta=8.85\left(\mathrm{~d}, J=5.2 \mathrm{~Hz}, 1 \mathrm{H}_{\text {arom }}\right), 8.65$ $\left(\mathrm{d}, J=8.8 \mathrm{~Hz}, 2 \mathrm{H}_{\text {arom }}\right), 8.26-8.23\left(\mathrm{~m}, 2 \mathrm{H}_{\text {arom }}\right), 7.75(\mathrm{~d}, J=8.0 \mathrm{~Hz}$, $\left.2 \mathrm{H}_{\text {arom }}\right), 7.61\left(\mathrm{~d}, J=5.6 \mathrm{~Hz}, 1 \mathrm{H}_{\text {arom }}\right), 7.56-7.54\left(\mathrm{~m}, 3 \mathrm{H}_{\text {arom }}\right), 7.39$ (t, $\left.J=8.0 \mathrm{~Hz}, 1 \mathrm{H}_{\text {arom }}\right), 7.29-7.21\left(\mathrm{~m}, 3 \mathrm{H}_{\text {arom }}\right), 3.89\left(\mathrm{~s}, 3 \mathrm{H}, \mathrm{OCH}_{3}\right)$.

${ }^{13} \mathrm{C} \mathrm{NMR}\left(400 \mathrm{MHz}, \mathrm{CDCl}_{3}\right): \delta=164.2,163.8,159.9,157.8(2 \mathrm{C})$, $143.1,142.1,136.9,130.9,129.8,128.9$ (2 C), 128.7 (2 C), 127.2 (2 C), 127.1 (2 C), 119.6, 114.4, 113.0, 112.8, 55.3.

HRMS (ESI): $m / z(\mathrm{M}+\mathrm{H})^{+}$calcd for $\mathrm{C}_{23} \mathrm{H}_{19} \mathrm{~N}_{2} \mathrm{O}: 339.1497$; found: 339.1503

\section{[3'-(4-Phenylpyrimidin-2-yl)biphenyl-3-yl]methanol (8d)}

Purification by column chromatography (20\% EtOAc-PE) gave 8d as an off-white solid; yield: $531 \mathrm{mg}(74 \%) ; \mathrm{mp} 123.1-125.7^{\circ} \mathrm{C}$.

${ }^{1} \mathrm{H}$ NMR (400 MHz, $\left.\mathrm{CDCl}_{3}\right): \delta=8.85\left(\mathrm{~d}, J=5.6 \mathrm{~Hz}, 1 \mathrm{H}_{\text {arom }}\right), 8.66$ $\left(\mathrm{d}, J=8.8 \mathrm{~Hz}, 2 \mathrm{H}_{\text {arom }}\right), 8.27-8.24\left(\mathrm{~m}, 2 \mathrm{H}_{\text {arom }}\right), 7.77(\mathrm{~d}, J=8.8 \mathrm{~Hz}$, $\left.2 \mathrm{H}_{\text {arom }}\right), 7.70\left(\mathrm{~s}, 1 \mathrm{H}_{\text {arom }}\right), 7.64-7.61\left(\mathrm{~m}, 2 \mathrm{H}_{\text {arom }}\right), 7.58-7.56(\mathrm{~m}, 3$ $\left.\mathrm{H}_{\text {arom }}\right), 7.48\left(\mathrm{t}, J=7.6 \mathrm{~Hz}, 1 \mathrm{H}_{\text {arom }}\right), 7.39\left(\mathrm{~d}, J=7.6 \mathrm{~Hz}, 1 \mathrm{H}_{\text {arom }}\right)$, $4.80\left(\mathrm{~s}, 2 \mathrm{H}, \mathrm{OCH}_{2}\right)$.

${ }^{13} \mathrm{C} \mathrm{NMR}\left(400 \mathrm{MHz}, \mathrm{CDCl}_{3}\right): \delta=163.9,163.5,157.3(2 \mathrm{C}), 142.7$, $141.1,140.4,136.4,136.3,130.6$ (2 C), 128.6, 128.5, 128.3, 126.8 (2 C), 126.0 (2 C), 125.8, 125.3 (2 C), 114.1, 64.8 .

HRMS (ESI): $m / z(\mathrm{M}+\mathrm{H})^{+}$calcd for $\mathrm{C}_{23} \mathrm{H}_{19} \mathrm{~N}_{2} \mathrm{O}: 339.1497$; found: 339.1504

\section{4-Phenyl-2-[4'-(trifluoromethyl)biphenyl-3-yl]pyrimidine (8e) Purification by column chromatography (15\% EtOAc-PE) gave 8e} as an off-white solid; yield: $679 \mathrm{mg}(85 \%)$; $\mathrm{mp} 188.1-190.3{ }^{\circ} \mathrm{C}$.

${ }^{1} \mathrm{H}$ NMR $\left(400 \mathrm{MHz}, \mathrm{CDCl}_{3}\right): \delta=8.88\left(\mathrm{~d}, J=5.2 \mathrm{~Hz}, 1 \mathrm{H}_{\text {arom }}\right), 8.70$ $\left(\mathrm{d}, J=8.4 \mathrm{~Hz}, 2 \mathrm{H}_{\text {arom }}\right), 8.27-8.25\left(\mathrm{~m}, 2 \mathrm{H}_{\text {arom }}\right), 7.81-7.73(\mathrm{~m}, 6$ $\left.\mathrm{H}_{\text {arom }}\right), 7.65\left(\mathrm{~d}, J=5.6 \mathrm{~Hz}, 1 \mathrm{H}_{\text {arom }}\right), 7.59-7.56\left(\mathrm{~m}, 3 \mathrm{H}_{\text {arom }}\right)$.

${ }^{13} \mathrm{C} \mathrm{NMR}\left(400 \mathrm{MHz}, \mathrm{CDCl}_{3}\right): \delta=164.0,163.9,157.8(2 \mathrm{C}), 144.1$, $141.7,137.7,136.8,131.0$ (2 C), 128.9 (2 C), 128.9 (2 C), 127.4 (2 C), 127.3 (2 C), 127.2 (2 C), 125.7, 125.7, 114.6.

HRMS (ESI): $m / z(\mathrm{M}+\mathrm{H})^{+}$calcd for $\mathrm{C}_{23} \mathrm{H}_{16} \mathrm{~F}_{3} \mathrm{~N}_{2}: 377.1266$; found: 377.1276

\section{2-(4'-Chlorobiphenyl-3-yl)-4-phenylpyrimidine (8f)}

Purification by column chromatography (10\% EtOAc-PE) gave $\mathbf{8 f}$ as an off-white solid; yield: $544 \mathrm{mg}(75 \%)$; $\mathrm{mp} 150.2-152.5^{\circ} \mathrm{C}$.

${ }^{1} \mathrm{H}$ NMR $\left(400 \mathrm{MHz}, \mathrm{CDCl}_{3}\right): \delta=8.87\left(\mathrm{~d}, J=5.2 \mathrm{~Hz}, 1 \mathrm{H}_{\text {arom }}\right), 8.68$ $\left(\mathrm{d}, J=8.4 \mathrm{~Hz}, 2 \mathrm{H}_{\text {arom }}\right), 8.27-8.25\left(\mathrm{~m}, 2 \mathrm{H}_{\text {arom }}\right), 7.72(\mathrm{~d}, J=8.4 \mathrm{~Hz}$, $\left.2 \mathrm{H}_{\text {arom }}\right), 7.63-7.56\left(\mathrm{~m}, 6 \mathrm{H}_{\text {arom }}\right), 7.45\left(\mathrm{~d}, J=8.0 \mathrm{~Hz}, 2 \mathrm{H}_{\text {arom }}\right)$.

${ }^{13} \mathrm{C} \mathrm{NMR}\left(400 \mathrm{MHz}, \mathrm{CDCl}_{3}\right): \delta=164.1,163.8,157.8$ (2 C), 141.9, 138.9, 137.1, 136.8, 133.7, 130.9 (2 C), 128.9, 128.9, 128.8, 128.7 (2 C), 128.3, 127.1 (2 C), 127.0 (2 C), 114.5.

HRMS (ESI): $m / z(\mathrm{M}+\mathrm{H})^{+}$calcd for $\mathrm{C}_{22} \mathrm{H}_{16} \mathrm{ClN}_{2}: 343.1002$; found: 343.1015

\section{2-[3-(2-Methoxypyridin-4-yl)phenyl]-4-phenylpyrimidine (8g)} Purification by column chromatography (25\% EtOAc-PE) gave $\mathbf{8 g}$ as an off-white solid; yield: $561 \mathrm{mg}(78 \%)$; $\mathrm{mp} 126.4-128.5^{\circ} \mathrm{C}$.

${ }^{1} \mathrm{H}$ NMR (400 MHz, $\left.\mathrm{CDCl}_{3}\right): \delta=8.85\left(\mathrm{~d}, J=5.6 \mathrm{~Hz}, 1 \mathrm{H}_{\text {arom }}\right), 8.66$ $\left(\mathrm{d}, J=8.4 \mathrm{~Hz}, 2 \mathrm{H}_{\text {arom }}\right), 8.49\left(\mathrm{~d}, J=2.4 \mathrm{~Hz}, 1 \mathrm{H}_{\text {arom }}\right), 8.25-8.23(\mathrm{~m}$, $\left.2 \mathrm{H}_{\text {arom }}\right), 7.89\left(\mathrm{~d}, J=8.8 \mathrm{~Hz}, 1 \mathrm{H}_{\text {arom }}\right), 7.68\left(\mathrm{~d}, J=8.4 \mathrm{~Hz}, 2 \mathrm{H}_{\text {arom }}\right)$, $7.61\left(\mathrm{~d}, J=5.6 \mathrm{~Hz}, 1 \mathrm{H}_{\text {arom }}\right), 7.56-7.54\left(\mathrm{~m}, 3 \mathrm{H}_{\text {arom }}\right), 6.86(\mathrm{~d}, J=8.8$ $\left.\mathrm{Hz}, 1 \mathrm{H}_{\text {arom }}\right), 4.0\left(\mathrm{~s}, 3 \mathrm{H}, \mathrm{OCH}_{3}\right)$.

${ }^{13} \mathrm{C} \mathrm{NMR}\left(400 \mathrm{MHz}, \mathrm{CDCl}_{3}\right): \delta=164.0,163.7,163.6,157.7$ (2 C), 145.0 (2 C), 139.8, 137.2, 136.7, 130.8, 129.3, 128.8 (2 C), 128.8 (2 C), 127.0 (2 C), 126.4 (2 C), 114.3, 110.7, 53.4.

HRMS (ESI): $m / z(\mathrm{M}+\mathrm{H})^{+}$calcd for $\mathrm{C}_{22} \mathrm{H}_{18} \mathrm{~N}_{3} \mathrm{O}$ : 340.1450 ; found: 340.1461 .

\section{2-(3',4'-Dichlorobiphenyl-3-yl)-4-phenylpyrimidine (8h)}

Purification by column chromatography (10\% EtOAc-PE) gave $\mathbf{8 h}$ as an off-white solid; yield: $606 \mathrm{mg}(76 \%)$; mp $121.3-123.7^{\circ} \mathrm{C}$.

${ }^{1} \mathrm{H} \mathrm{NMR}\left(400 \mathrm{MHz}, \mathrm{CDCl}_{3}\right): \delta=8.86\left(\mathrm{~d}, J=5.2 \mathrm{~Hz}, 1 \mathrm{H}_{\text {arom }}\right), 8.66$ $\left(\mathrm{d}, J=8.4 \mathrm{~Hz}, 2 \mathrm{H}_{\text {arom }}\right), 8.26-8.23\left(\mathrm{~m}, 2 \mathrm{H}_{\text {arom }}\right), 7.76(\mathrm{~d}, J=2.0 \mathrm{~Hz}$, $\left.1 \mathrm{H}_{\text {arom }}\right), 7.69\left(\mathrm{~d}, J=8.4 \mathrm{~Hz}, 2 \mathrm{H}_{\text {arom }}\right), 7.64\left(\mathrm{~d}, J=5.2 \mathrm{~Hz}, 1 \mathrm{H}_{\text {arom }}\right)$, 7.56-7.51 (m, $\left.5 \mathrm{H}_{\text {arom }}\right)$.

${ }^{13} \mathrm{C} \mathrm{NMR}\left(400 \mathrm{MHz}, \mathrm{CDCl}_{3}\right): \delta=164.1,163.9,157.6(2 \mathrm{C}), 155.3$, $140.7,140.5,137.4,136.6,132.9,131.7,131.1,130.7,130.7$ (2 C), $128.9,128.9,128.9,127.2,127.0,126.3(2 \mathrm{C})$.

HRMS (ESI): $m / z(\mathrm{M}+\mathrm{H})^{+}$calcd for $\mathrm{C}_{22} \mathrm{H}_{15} \mathrm{Cl}_{2} \mathrm{~N}_{2}: 377.0612$; found: 377.0624 .

\section{Acknowledgment}

The authors thank Dr. A. K. Roy, Dr. K. V. Raghu, Dr. V. Dahanukar, and the analytical group of Dr. Reddy's Laboratories, Hyderabad, India for spectral data, and CSIR, Human Resources Development Group, Govt. of India, New Delhi for providing financial assistance [No. 01(2391)/10/EMR-II].

Supporting Information for this article is available online at http://www.thieme-connect.com/ejournals/toc/synthesis.

\section{References}

(1) The term 'privileged scaffolds or structures' was originally introduced by Merck researchers in their work on benzodiazepines: (a) Evans, B. E.; Rittle, K. E.; Bock, M. G.; DiPardo, R. M.; Freidinger, R. M.; Whitter, W. L.; Lundell, G. F.; Veber, D. F.; Anderson, P. S. J. Med. Chem. 1988, 31, 2235. (b) Patchett, A. A.; Nargund, R. P. Ann. Rep. Med. Chem. 2000, 35, 289.

(2) (a) Press, J. B.; McNally, J. J.; Keiser, J. A.; Offord, S. J.; Katz, L. B.; Giardino, E.; Falotico, R.; Tobia, A. J. Eur. J. Med. Chem. 1989, 24, 627. (b) Alam, O.; Khan, S. A.; Siddiqui, N.; Ahsan, W.; Verma, S. P.; Gilani, S. J. Eur. J. Med. Chem. 2010, 45, 5113. (c) Amin, K. M.; Awadalla, F. M.; Eissa, A. A. M.; Abou-Seri, S. M.; Hassan, G. S. Bioorg. Med. Chem. 2011, 19, 6087.

(3) (a) Bruno, O.; Schenone, S.; Ranise, A.; Barocelli, E.; Chiavarini, M.; Ballabeni, V.; Bertoni, S. Arzneim.Forsch./Drug Res. 2000, 50, 140. (b) Bruno, O.; Brullo, C.; Schenone, S.; Bondavalli, F.; Ranise, A.; Tognolini, M.; Ballabeni, V.; Barocelli, E. Bioorg. Med. Chem. 2004, 12, 553.

(4) Wyrzykiewicz, E.; Bartkowiak, G.; Kedzia, B. Farmaco 1993, 48, 979.

(5) Sharma, P.; Rane, N.; Gurram, V. K. Bioorg. Med. Chem. Lett. 2004, 14, 4185.

(6) Elkholy, Y. M.; Morsy, M. A. Molecules 2006, 11, 890.

(7) Holla, B. S.; Mahalinga, M.; Karthikeyan, M. S.; Akberali, P. M.; Shetty, N. S. Bioorg. Med. Chem. 2006, 14, 2040.

(8) Ingarsal, N.; Saravanan, G.; Amutha, P.; Nagarajan, S. Eur. J. Med. Chem. 2007, 42, 517.

(9) Zhao, X.-L.; Zhao, Y.-F.; Guo, S.-C.; Song, H.-S.; Wang, D.; Gong, P. Molecules 2007, 12, 1136. 
(10) Cordeu, L.; Cubedo, E.; Bandrés, E.; Rebollo, A.; Sáenz, X.; Chozas, H.; Domínguez, M. V.; Echeverría, M.; Mendivil, B.; Sanmartin, C.; Palop, J. A.; Font, M.; García-Foncillas, J. Bioorg. Med. Chem. 2007, 15, 1659.

(11) Sondhi, S. M.; Singh, N.; Johar, M.; Kumar, A. Bioorg. Med. Chem. 2005, 13, 6158.

(12) Amin, K. M.; Hanna, M. M.; Abo-Youssef, H. E.; George, R. F. Eur. J. Med. Chem. 2009, 44, 4572.

(13) (a) Baumgarth, M.; Beier, N.; Gericke, R. J. Med. Chem. 1997, 40, 2017. (b) Ramesh, L. S.; Varsha, I. S.; Ganesh, D. J.; Jyoti, B. W. Med. Chem. Res. 2012, 21, 1825.

(14) Nega, S.; Aionso, J.; Diazj, A.; Junquere, F. J. Heterocycl. Chem. 1990, 27, 269.

(15) Shishoo, C. J.; Jain, K. S. J. Heterocycl. Chem. 1992, 29, 883.

(16) Peters, J. U.; Hunziker, D.; Fischer, H.; Kansy, M.; Weber, S.; Kritter, S.; Muller, A.; Ricklin, F.; Boehringer, M.; Poli, S. M.; Csato, M.; Loeffler, B. M. Bioorg. Med. Chem. Lett. 2004, 14, 3575 .

(17) Peters, J. U.; Weber, S.; Kritter, S.; Weiss, P.; Wallier, A.; Zimmerli, D.; Boehringer, M.; Steger, M.; Loeffler, B. M. Bioorg. Med. Chem. Lett. 2004, 14, 3579.

(18) For reviews, see: (a) Undheim, K.; Benneche, T. In Comprehensive Heterocyclic Chemistry II; Vol. 6; Katritzky, A. R.; Rees, C. W.; Scriven, E. F. V.; McKillop, A., Eds.; Pergamon: Oxford, 1996, 93. (b) Lagoja, I. M. Chem. Biodiversity 2005, 2, 1. (c) Michael, J. P. Nat. Prod. Rep. 2005, 22, 627. (d) Joule, J. A.; Mills, K. I. Heterocyclic Chemistry, 4th ed.; Blackwell Science Ltd: Cambridge,
2000, 194. (e) Hill, M. D.; Movassaghi, M. Chem.-Eur. J. 2008, 14, 6836 .

(19) For reviews, see: (a) Turck, A.; Ple, N.; Mongin, F.; Queguiner, G. Tetrahedron 2001, 57, 4489. (b) Chinchilla, R.; Najera, C.; Yus, M. Chem. Rev. 2004, 104, 2667. (c) Schroder, S.; Stock, C.; Bach, T. Tetrahedron 2005, 61, 2245. (d) Martin, R.; Buchwald, S. L. Acc. Chem. Res. 2008, 41, 1461. (e) Surry, D. S.; Buchwald, S. L. Angew. Chem. Int. Ed. 2008, 47, 6338.

(20) Passerini three-component and Ugi four-component condensations are the most popular among many other reactions for their wide scope and synthetic utility. For reviews, see: (a) Bienayme, H.; Hulme, C.; Oddon, G.; Schmitt, P. Chem. Eur. J. 2000, 6, 3321. (b) Dömling, A.; Ugi, I. Angew. Chem. Int. Ed. 2000, 39, 3168.

(21) For recent reviews, see: (a) Toure, B. B.; Hall, D. G. Chem. Rev. 2009, 109, 4439. (b) Balme, G.; Bossharth, E.;

Monteiro, N. Eur. J. Org. Chem. 2003, 4101. (c) Hulme, C.; Gore, V. Curr. Med. Chem. 2003, 1051. (d) Zhu, J. Eur. J. Org. Chem. 2003, 1133.

(22) Weber, L.; Illgen, K.; Almstetter, M. Synlett 1999, 366.

(23) (a) Lin, Y-i.; Lang, S. A. Jr. J. Heterocycl. Chem. 1977, 14, 345. (b) Bredereck, H.; Effenberger, F.; Botsch, H. Chem. Ber. 1964, 97, 3397. (c) Junek, H.; Schmidt, A. Monatsh. Chem. 1968, 99, 635. (d) Junek, H.; Stolz, G. Monatsh. Chem. 1970, 201, 1234.

(24) Medwid, J. B.; Rolf, P.; Baker, J. S.; Brockman, J. A.; Du, M. T.; Hallett, W. A.; Hanifin, W. J.; Hardy, R. A.; Ernestine, T. M. Jr.; Torley, L. W.; Wren, S. J. Med. Chem. 1990, 33, 1230. 